\title{
THE NET REPRODUCTIVE VALUE AND STABILITY IN MATRIX POPULATION MODELS
}

\author{
J.M. CUSHING \\ Department of Mathematics \\ Interdisciplinary Program on Applied Mathematics \\ University of Arizona \\ Tucson, AZ 85721 \\ e-mail: cushing@math.arizona.edu \\ ZHOU YICANG \\ Department of Mathematics \\ Xi'an Jiaotong University \\ Xi'an, Shaanxi 710049 \\ China
}

\begin{abstract}
The net reproductive value $n$ is defined for a general discrete linear population model with a nonnegative projection matrix. This number is shown to have the biological interpretation of the expected number of offspring per individual over its life time. The main result relates $n$ to the population's growth rate (i.e. the dominant eigenvalue $\lambda$ of the projection matrix) and shows that the stability of the extinction state (the trivial equilibrium) can be determined by whether $n$ is less than or greater than 1. Examples are given to show that explicit algebraic formulas for $n$ are often derivable, and hence available for both numerical and parameter studies of stability, when no such formulas for $\lambda$ are available.
\end{abstract}

1. Introduction. Fertility rates are among the most important vital parameters in population studies. Different measures of fertility, such as age-specific fertility rates or the total fertility rate (e.g. see Newell [1988]), have been used in demography and mathematics to describe the influence of births on a population's dynamics. In applied mathematical demography and in age-structured population dynamics the net reproductive value (often called the net reproductive rate or number) plays an important central role. It combines together the age-specific fertility rates and the age-specific survival (or mortality) rates and gives the expected number of offsprings per individual over its life time. It has been introduced and widely used in age-structured

Research of the first author supported by NSF grant \# DMS-9319073. 
population models for more than one hundred years Caswell [1989], Keyfitz [1985], and Smith and Keyfitz [1977]. It has been used to characterize the stability of the trivial equilibrium and as a bifurcation parameter in the study of positive equilibrium for linear and nonlinear models (Cushing [1988, 1993, 1994] and Jian and Jingyuan [1988]).

In this paper we show mathematically how to define the net reproductive value $n$ for a very general class of matrix population models. Our goal is to show that this quantity is both biologically meaningful and analytically useful. The general definition is given in Section 2. Theorem 3 gives a relationship between $n$ and the population growth rate $\lambda$ (i.e. the dominant eigenvalue of the projection matrix, or the so-called Perron root). The exact relationship between $n$ and the dynamic stability of linear models is given in Corollary 4. This stability result is a generalization of that for linear, Leslie age-structured population models. In Section 3 several examples are given to show how to apply the net reproductive value to determine stability. These applications concentrate on the calculation of $n$ and show some advantages over the calculation $\lambda$. For example, under certain assumptions an algebraic formula for the net reproductive value $n$ is derived for general Lefkovitch [1965] models. This includes a formula for any demographic model in which all newborns lie in a single class, such as the Leslie and Usher models. Although the net reproductive value is defined for linear structured population models, we show in Section 4 how it can also be used in the study of some nonlinear structured population models by means of linearization techniques.

2. The net reproductive value and stability. The asymptotic dynamics of a linear population model are determined, under minimal conditions, by the dominant eigenvalue $\lambda>0$ of the projection matrix. If this number is less than 1 then the population dies out asymptotically or, in other words, the zero state equilibrium is globally stable. On the other hand, if $\lambda$ is greater than 1 then the population will grow exponentially and the zero equilibrium will be unstable. For agestructured matrix models with Leslie projection matrices these facts are well known. However, they remain valid (by the famous PerronFrobenius theory) for general non-negative projection matrices under some restrictive, but still quite general assumptions.

For Leslie models another parameter can also be used to determine 
the stability properties of the population, namely the net reproductive value $n$. This number and its relationship to the dynamics of the population are discussed in the following Section 2.1. One advantage of the number $n$ is that a simple algebraic formula is available for it in terms of the entries in the Leslie matrix (see (2) and (14) below). This formula permits studies to be carried out on the relationship between model parameters (age specific birth rates, death rates, etc.) and the long term dynamics of the population. Also, it allows for the easy numerical calculation of $n$ when model parameters are assigned numerical values. There is no such algebraic formula for the dominant eigenvalue $\lambda$.

In Section 2.2 we give a mathematical definition of the "net reproductive value" $n$ for a general class of matrix population models and show that it equal to the expected number of offspring per individual over its lifetime. In Section 2.3 our main results relating $n$ to the dominant eigenvalue $\lambda$ and hence to the asymptotic dynamics of the population are given in Theorem 3 and its Corollary 4 . Formulas for $n$ are given for some general models, including those with a single reproductive class (such as Leslie and Usher models).

2.1. Age-structured population models. Matrix models have been used by many authors to study age-structured populations; e.g. see Caswell [1989], Impagliazzo [1985], Lewis [1942], Leslie [1948] and Newell [1988]. Let $x_{i}(t), i=1,2, \ldots, m$, denote the number (or density) of individuals in the $i^{\text {th }}$ age class at time $t$, and suppose that the time unit is taken, without loss in generality, to be 1 . Let $p_{i+1, i} \in(0,1)$ be the fraction of individuals in age class $i$ that survives to age class $i+1$ after one time unit. Let $f_{1 i}, i=1,2, \ldots, m$, be the number of offsprings produced by an individual in age class $i$ that survives to age 1 . Then the discrete age-structured population model is

$$
\begin{aligned}
x_{1}(t+1) & =\sum_{j=1}^{m} f_{1 j} x_{j}(t) \\
x_{i+1}(t+1) & =p_{i+1, i} x_{i}(t), \quad i=1,2, \ldots, m-1
\end{aligned}
$$

for

$$
t=t_{0}, t_{0}+1, t_{0}+2, \ldots
$$


Given an initial population distribution $x_{i}\left(t_{0}\right)=x_{i}^{0} \geq 0, i=$ $1,2, \ldots, m$, then the dynamics of the population are uniquely determined by these formulas for all $t>t_{0}$.

In this model the net reproductive value is defined as

$$
n=\sum_{i=1}^{m} f_{1 i} \prod_{j=0}^{i-1} p_{j+1, j}
$$

where, for notational convenience only, we denote $p_{10}=1$. The number defined by (2) has a straightforward biological meaning. The product $\prod_{j=0}^{i-1} p_{j+1, j}$ is the probability that an individual lives to age $i$ and the product $f_{1 i} \prod_{j=0}^{i-1} p_{j+1, j}$ is the number of its offspring. Consequently, $n$ is the expected number of offspring per individual over its life time.

Let

$$
\mathbf{x}(t)=\left(\begin{array}{c}
x_{1}(t) \\
x_{2}(t) \\
\vdots \\
x_{m}(t)
\end{array}\right)
$$

denote the age-distribution vector at time $t$. We have following lemma (Cushing [1988]).

LEMMA 1. For any initial distribution $\mathbf{x}\left(t_{0}\right)=\mathbf{x}^{0} \geq 0(\neq 0)$

(i) $n<1$ implies $\lim _{t \rightarrow \infty} x_{i}(t)=0$ for all $i=1,2, \ldots, m$

(ii) $n>1$ implies $\lim _{t \rightarrow \infty} x_{i}(t)=+\infty$ for all $i=1,2, \ldots, m$.

If $n=1$ then there exist positive equilibrium solutions $\mathbf{x}=c \mathbf{v}$ of the model equations (1) where $c$ is an arbitrary positive constant and

$$
0<\mathrm{v}=\left(\begin{array}{c}
1 \\
p_{21} \\
p_{21} p_{32} \\
p_{21} p_{32} p_{43} \\
\vdots \\
p_{21} p_{32} \cdots p_{m, m-1}
\end{array}\right) .
$$

Lemma 1 illustrates the role of the net reproductive value $n$ in determining the asymptotic dynamics of the population. The trivial 
solution $\mathbf{x}=0$ of equations (1) is asymptotically stable if $n<1$ and is unstable if $n>1$. If $n=1$, nontrivial equilibria exist. The critical value $n=1$ is a bifurcation value in the sense that when $n$ passes through 1 the stability of the trivial solution changes. At $n=1$ a continuum of positive equilibrium solutions of equations (1) bifurcates from the trivial equilibrium. Biologically, $n<1$ means that an individual cannot fully replace itself during its life span and therefore the population decreases. In the case $n>1$, the situation is the opposite. When $n=1$ an individual produces exactly one offspring to replace itself over its lifetime and, as a result, the whole population maintains itself at a constant level.

2.2. General structured models. Structured population models designate classifications or cohorts of individuals and follow these cohorts in time. The classes or stages are defined according to what properties of an individual are most important so far as its vital rates are concerned. The most common classifications are chronological age, body size, etc. One of the major goals of structured population dynamics is to bridge the gap between the level of the individual organism and the level of the population as a whole. This will allow the mathematical models to take better into account the vast amount of knowledge and data that biologists have amassed about individual biological organisms and to make them more accurate and predictive.

In Leslie age-structured population models offspring are born into the first age class only and individuals in age-class $i$ at time $t$ can only move to age class $i+1$ at time $t+1$. In generalized structured population models these two restrictions are relaxed. Offspring may be born into any class and individuals in class $i$ at time $t$ can transfer to any class $j$ at time $t+1$ (Caswell [1989], Cushing [1988, 1993], Lefkovitch [1965]).

We will consider here only linear autonomous structured models for populations closed to immigration and emigration. Suppose that the individuals of a population are categorized into $m$ classes. The number (or density) $x_{i}(t)$ in class $i$ at time $t$ is placed in a class distribution vector $\mathbf{x}(t)$. Let $p_{i j} \in[0,1]$ be the fraction of individuals in class $j$ expected to transfer to class $i$ during one unit time. It is clear that $0 \leq \sum_{i=1}^{m} p_{i j} \leq 1$. Let $T$ denote the $m \times m$ "transition" matrix 
consisting of these transition probabilities, i.e.

$$
\begin{gathered}
T=\left(p_{i j}\right), \quad p_{i j} \in[0,1] \\
0 \leq \sum_{i=1}^{m} p_{i j} \leq 1, \quad j=1,2, \ldots, m .
\end{gathered}
$$

The individuals in the population at time $t$, distributed according to the vector $\mathbf{x}(t)$, who survive to time $t+1$ will then be redistributed according to the vector $T \mathbf{x}(t)$.

Let $f_{i j}$ denote the expected number of $i$-class newborns per $j$-class individual alive at time $t+1$ due to births during the time interval $(t, t+1)$. Let $F$ be the $m \times m$ "production" (or "fertility") matrix

$$
F=\left(f_{i j}\right) \geq 0, \quad f_{i j} \geq 0 \quad \sum_{i, j=1}^{m} f_{i j} \neq 0 .
$$

The distribution vector of newborns at time $t+1$ due to births from individuals of the class distribution vector $\mathbf{x}(t)$ at time $t$ is $F \mathbf{x}(t)$. With these notations the distribution $\mathbf{x}(t+1)$ at time $t+1$ is given by the matrix difference equation system

$$
\begin{gathered}
\mathbf{x}(t+1)=A \mathbf{x}(t), \quad \mathbf{x}\left(t_{0}\right) \geq 0 \\
t=t_{0}, t_{0}+1, t_{0}+2, \ldots
\end{gathered}
$$

where the coefficient matrix $A$, usually referred to as the "projection matrix," is given by

$$
A=F+T \geq 0 \text {. }
$$

It is obvious that $\mathbf{x}(t)$ is uniquely determined for all $t>t_{0}$ by recursive formula (5) once the initial distribution vector $\mathbf{x}\left(t_{0}\right)$ is given.

We assume that

(7) the matrix A given by (6), (4), and (3) has a positive, simple, strictly dominant eigenvalue $\lambda$ whose associated (right) eigenvector $\mathrm{v}>0$ is strictly positive. 
Sufficient for this assumption is that $A$ is reducible and primitive (which is equivalent to an integer power $A^{n}>0$ of $A$ being strictly positive) (Gantmacher [1959]).

The first step in understanding the dynamics of a model population described by the equation (5) is to determine the equilibrium (i.e. constant) solutions and the stability of the equilibrium. It is clear that $\mathrm{x}=0$ is an equilibrium. Is the trivial equilibrium 0 stable? Are there any positive equilibria? It is natural to ask if it is possible to define, as was done for the Leslie age-structured population model (1), a "net reproductive value" $n$ for the general structured population model (5) which determines the stability of the trivial equilibrium 0 and the bifurcation of positive equilibrium. We will now show how to define such a number under two assumptions on the matrices $F$ and $T$.

First, we assume that the inverse

$$
(I-T)^{-1}=\left(e_{i j}\right) \text { exists. }
$$

A necessary condition for this is that at least one of the column sums $\sum_{i=1}^{m} p_{i j}$ of $T$ be strictly less than 1 . A sufficient condition is that all of the column sums of $T$ be strictly less than 1 (i.e. that there is some loss to each class over each time interval)

$$
0 \leq \sum_{i=1}^{m} p_{i j}<1, \quad j=1,2, \ldots, m
$$

The entry $e_{i j}$ is the expected amount of time that will be spent in class $i$ by an individual starting in class $j$ over the course of its life time. The entry $r_{i j}$ in the matrix $(I-T)^{-1} F=\left(r_{i j}\right)$ gives the expected number of $i$ class offspring that an individual born into class $j$ will produce over its life time. Our second assumption is that

(10) $(I-T)^{-1} F$ has a positive, simple, strictly dominant eigenvalue $n$ whose right eigenvector $\mathrm{y}>0$ is strictly positive.

Sufficient for this assumption is that $(I-T)^{-1} F$ is non-negative, irreducible and primitive or, equivalently, that some integer power of $(I-T)^{-1} F \geq 0$ is strictly positive. (This is not necessary, however, 
since we do not require that the left eigenvalue be strictly positive; see the application in Section 4.2 below.) Assumption (9) implies that $(I-T)^{-1}=\sum_{k=0}^{\infty} T^{k}$ and hence that $(I-T)^{-1} \geq 0$.

DEFINITION 2. Assume that $F$ and $T$ satisfy (8) and (10). Then $n$ is called the net reproductive value for the model equation (5)-(6).

This definition of net reproductive value is, of course, purely mathematical. A natural question to ask is whether in fact it has the correct biological interpretation. That is to say, is $n$ equal to the expected number of offspring produced by an individual over its life time? In age-structured population (Leslie) models the newborns are always in the first age class and after each time unit surviving individuals advance into the next age class. Therefore, it is easy to trace a newborn cohort in time and to record its reproductive activity. In this case, the formula (2) for $n$ in terms of the age specific fertility and survival rates can be straightforwardly obtained and the fact that $n$ is the expected number of offspring per individual over its life time is clear. In the general structured model (5) newborns can lie in any of the classes and individuals in one class can transfer to any other class in one unit of time. In this case it is not as easy to trace an individual's progression through the different classes. Here we will consider one case only. Let us follow one cohort in time and calculate the average number of offspring over the expected life time of the cohort. Specifically, consider the newborn cohort produced by an adult population whose distribution is $\mathbf{y}>0$, the positive eigenvector corresponding to $n$. For simplicity assume (9). From

$$
(I-T)^{-1} F \mathbf{y}=n \mathbf{y}
$$

or

$$
F \mathbf{y}=n(I-T) \mathbf{y}
$$

we see that from the parent distribution $\mathbf{y}$ the distribution $\mathbf{z}=F \mathbf{y}$ of the newborn cohort is

$$
\mathbf{z}=n(I-T) \mathbf{y} .
$$

After $k$ time steps the distribution of this cohort $\mathbf{z}$ is $T^{k} \mathbf{z}, k=$ $0,1,2, \ldots$, and the offspring produced by this cohort is $F T^{k} \mathbf{z}, k=$ $0,1,2, \ldots$ The distribution of the total expected number of the 
offspring produced by the cohort $\mathbf{z}$ is

$$
\sum_{k=0}^{\infty} F T^{k} \mathbf{z}=F(I-T)^{-1} \mathbf{z}=n \mathbf{z} .
$$

Therefore, the expected number of offspring per individual over its life time is equal to

$$
\frac{\sum_{i=1}^{m} n z_{i}}{\sum_{i=1}^{m} z_{i}}=n .
$$

That is to say, the expected number of offspring per individual over its life time is the same, by our definition, as the net reproductive value.

As an example, for two-dimensional structured population models defined by

$$
F=\left(\begin{array}{ll}
f_{11} & f_{12} \\
f_{21} & f_{22}
\end{array}\right), \quad T=\left(\begin{array}{ll}
p_{11} & p_{12} \\
p_{21} & p_{22}
\end{array}\right)
$$

the net reproductive value is given by the formula

$$
\begin{array}{ll}
n=\frac{1}{2} \frac{1}{\left(1-p_{11}\right)\left(1-p_{22}\right)-p_{12} p_{21}}\left(\alpha+\beta+\left((\alpha-\beta)^{2}+4 \gamma \delta\right)^{1 / 2}\right) \\
\alpha=p_{12} f_{21}+\left(1-p_{22}\right) f_{11}, \quad \beta=p_{21} f_{12}+\left(1-p_{11}\right) f_{22} \\
\gamma=p_{12} f_{22}+\left(1-p_{22}\right) f_{12}, \quad \delta=p_{21} f_{11}+\left(1-p_{11}\right) f_{22} .
\end{array}
$$

As another example, the net reproductive value is given by the formula (2) for age-structured Leslie models (1). Formulas for other types of projection matrices are given below.

2.3. The net reproductive value and stability. The linear autonomous structured population model (5) has the unique solution

$$
\mathbf{x}(t)=A^{t} \mathbf{x}(0), \quad t=0,1,2, \ldots .
$$

It is obvious that $\mathbf{x}=0$, the trivial solution, is an equilibrium of model (5). By using the Jordan canonical form it is easy to see that the stability of $\mathrm{x}=0$ depends upon the eigenvalues of the non-negative matrix $A$. Stability and bifurcation of the equilibria for model (5) are then determined by the dominant eigenvalue $\lambda$ (Caswell [1989]). 
In age-structured population models the net reproductive value $n$ determines the stability of the trivial equilibrium. For generalized structured population models the net reproductive value is a generalization of that for age structured population models. Therefore, it is natural to conjecture that the net reproductive value determines the stability of the trivial equilibrium in the general model as well (Cushing [1993]). As the following theorem shows this conjecture is true under assumptions (7), (8) and (10).

THEOREM 3. Assume that $A$ satisfies (7) and that $F$ and $T$ satisfy (8) and (10). Then $\lambda>1$ (respectively $\lambda<1$ or $\lambda=1$ ) if and only if $n>1$ (respectively $n<1$ or $n=1$ ).

This theorem can be proved for two-dimensional matrices $A$ or for arbitrary age-structured population matrices by a straightforward analysis of the dominant eigenvalue and the formulas for the net reproductive value given above. A proof for the general case is given in the Appendix.

From Theorem 3 we have following stability result.

COROLLARY 4. Under the conditions of Theorem 3, the trivial equilibrium $\mathrm{x}=0$ of model (5) is asymptotically stable (respectively unstable) if and only if $n<1$ (respectively $n>1$ ). If $n=1$ then there exist a family of positive equilibria $\mathbf{x}=c \mathbf{v}$ where $c$ is an arbitrary positive constant.

3. Applications to linear models In this section the net reproductive value $n$ will be examined for several linear matrix population models. They will demonstrate the usefulness of using $n$ in studying the asymptotic dynamics of the population. We will derive an algebraic formula that can be used to calculate $n$ from the entries of the projection matrix $A=F+T$ in the case when all newborns lie in a single class (see (19)). This includes, of course, Leslie and Usher models. No such formula is available for $\lambda$ and this quantity can only be calculated, by some numerical eigenvalue procedure, when numerical values are assigned to the entries in $A$.

We point out that in the case considered here, when all newborns lie in a single class, the "mean generation time" $\gamma$ is given by the formula 
$\gamma=\ln n / \ln \lambda($ Caswell [1989]).

3.1. Leslie models. Consider the model equation (5)-(6) in which

$$
\begin{gathered}
f=\left(\begin{array}{cccc}
f_{11} & f_{12} & \cdots & f_{1 m} \\
0 & 0 & \cdots & 0 \\
\vdots & \vdots & \square & \vdots \\
0 & 0 & \cdots & 0
\end{array}\right) \\
T=\left(\begin{array}{ccccccc}
0 & 0 & 0 & \cdots & 0 & 0 & 0 \\
p_{21} & 0 & 0 & \cdots & 0 & 0 & 0 \\
0 & p_{32} & 0 & \cdots & 0 & 0 & 0 \\
\vdots & \vdots & \vdots & \square & \vdots & \vdots & \vdots \\
0 & 0 & 0 & \cdots & p_{m-1, m-2} & 0 & 0 \\
0 & 0 & 0 & \cdots & 0 & p_{m, m-1} & p_{m m}
\end{array}\right)
\end{gathered}
$$

with

$$
0<p_{i+1,1}<1, \quad 0 \leq p_{m m}<1 .
$$

Under these conditions, the transition matrix $T$ satisfies the condition (9) and hence (8) holds. By direct calculation

$$
(I-T)^{-1} F=\left(\begin{array}{llll}
f_{11} \mathbf{y} & f_{12} \mathbf{y} & \cdots & f_{1 m} \mathbf{y}
\end{array}\right)
$$

where

$$
0<\mathbf{y}=\left(\begin{array}{c}
1 \\
p_{21} \\
p_{21} p_{32} \\
\vdots \\
p_{21} p_{32} \cdots p_{m-1, m-2} \\
\left(1-p_{m m}\right)^{-1} \Pi_{k=0}^{m-1} p_{k+1, k}
\end{array}\right)
$$

Here, for notational convenience, we have set $p_{10}=1$. Because all of its columns are proportional to $\mathrm{y}$ the matrix $(I-T)^{-1} F$ has 0 as an eigenvalue of multiplicity $m-1$. The remaining eigenvalue is

$$
n=\sum_{j=1}^{m-1} f_{1 j} \prod_{k=0}^{j-1} p_{k+1, k}+\frac{p_{21} p_{32} \cdots p_{m, m-1}}{1-p_{m m}} f_{1 m}>0 .
$$


The positive vector $\mathrm{y}$ is a (right) eigenvector of $(I-T)^{-1} \mathrm{~F}$ associated with $n$. Because $n$ is positive and strictly dominant this shows that the Leslie model satisfies (10) and that Theorem 3 and its Corollary 4 apply. Thus, the trivial equilibrium $\mathbf{x}=0$ is stable (unstable) if and only if $n<1(n>1)$.

As an specific numerical example, consider the case when

$$
A=\left(\begin{array}{ccccccc}
0 & 0 & 0.19 & 0.44 & 0.5 & 0.5 & 0.45 \\
0.87 & 0 & 0 & 0 & 0 & 0 & 0 \\
0 & 0.87 & 0 & 0 & 0 & 0 & 0 \\
0 & 0 & 0.87 & 0 & 0 & 0 & 0 \\
0 & 0 & 0 & 0.87 & 0 & 0 & 0 \\
0 & 0 & 0 & 0 & 0.87 & 0 & 0 \\
0 & 0 & 0 & 0 & 0 & 0.87 & 0.80
\end{array}\right)
$$

which was studied by Usher as a model for blue whale populations (the time interval being two years) (Usher [1972]). From equation (14) we calculate $n \simeq 1.9494>1$. Corollary 4 implies that $\mathbf{x}=0$ is unstable and hence that the population is growing exponentially.

From Corollary 7 of the Appendix we know that $1<\lambda<n \simeq 1.9494$. In fact, the dominant eigenvalue $\lambda$ of the projection matrix $A$ can be approximated numerically (as a root of the sixth degree characteristic polynomial or by other eigenvalue methods) to be $\lambda \simeq 1.0986$. This is the exponential growth rate of the population. The mean length of a generation is then $\gamma=\ln n / \ln \lambda \simeq 7.0985$ time units, or 14.197 years.

3.2. Usher models. Usher matrices are a slightly more general kind of projection matrices than Leslie matrices. They have found application in models based upon size structuring as opposed to chronological age (Caswell [1989], Usher [1966, 1969, 1972]). In an Usher model the projection matrix $A=F+T$ has the same fertility matrix $F$ as that in a Leslie matrix, namely (12), and a transition matrix that is a Leslie matrix (13) with added transition probabilities $p_{i i} \in[0,1]$, down the 
main diagonal, i.e.

(15) $\quad T=\left(\begin{array}{ccccccc}p_{11} & 0 & 0 & \cdots & 0 & 0 & 0 \\ p_{21} & p_{22} & 0 & \cdots & 0 & 0 & 0 \\ 0 & p_{32} & p_{33} & \cdots & 0 & 0 & 0 \\ \vdots & \vdots & \vdots & \square & \vdots & \vdots & \vdots \\ 0 & 0 & 0 & \cdots & p_{m-1, m-2} & p_{m-1, m-1} & 0 \\ 0 & 0 & 0 & \cdots & 0 & p_{m, m-1} & p_{m m}\end{array}\right)$.

Usher used this projection matrix to model forest dynamics where $p_{i i}$ is the probability that a tree in the $i^{i t}$ size class will remain in that size class after a time unit, $p_{i+1 i}$ is the probability that a tree in the $i^{\text {th }}$ size class will grow into the $(i+1)^{t h}$ size class after a time unit, and $f_{1 i}$ is the rate of the regeneration from the $i^{\text {th }}$ class. The conditions Usher assumed are

$$
\begin{aligned}
& 0 \leq p_{i i}<1 \text { and } f_{1 i} \geq 0 \text { for } i=1,2, \ldots, m \\
& 0<p_{i+1, i} \text { and } p_{i i}+p_{i+1, i} \leq 1 \text { for } i=1,2, \ldots, m-1
\end{aligned}
$$

Note that the dissipation condition (9) may not hold. Nonetheless, the conditions (8) and (10) required for Theorem 3 and Corollary 4 are fulfilled, as we will now show. A straightforward calculation shows that $(I-T)^{-1}$ exists and that

$$
(I-T)^{-1} F=\left(\begin{array}{llll}
f_{11} \mathrm{y} & f_{12} \mathrm{y} & \cdots & f_{1 m} \mathrm{y}
\end{array}\right)
$$

where

$$
\mathrm{y}=\left(\begin{array}{c}
\frac{1}{1-p_{11}} \\
\frac{p_{21}}{\left(1-p_{11}\right)\left(1-p_{22}\right)} \\
\vdots \\
\frac{\prod_{k=1}^{m} p_{k, k-1}}{\prod_{k=1}^{m}\left(1-p_{k k}\right)}
\end{array}\right)>0
$$

As in the Leslie matrix case, $(I-T)^{-1} F$ has 0 as an eigenvalue of multiplicity $m-1$. The remaining, positive and strictly dominant, eigenvalue is

$$
n=\sum_{i=1}^{m} f_{1 i} \prod_{k=1}^{i} \frac{p_{k, k-1}}{1-p_{k k}}
$$


The positive vector $\mathrm{y}$ is a (right) eigenvector of $(I-T)^{-1} \mathrm{~F}$ associated with $n$.

We have an algebraic formula for the net reproductive value which, by Theorem 3 and Corollary 4, can be used to determine the stability of the trivial equilibrium and hence the growth and survival or the decay and ultimate extinction of the population. Thus, it is a simple matter in numerical applications to calculate $n$. This is in contrast to the calculation of the dominant eigenvalue $\lambda$ of the projection matrix.

Here is a numerical example. The matrix

$$
A=\left(\begin{array}{cccccc}
0.72 & 0 & 0 & 0.74 & 1.04 & 9.03 \\
0.28 & 0.69 & 0 & 0 & 0 & 0 \\
0 & 0.31 & 0.75 & 0 & 0 & 0 \\
0 & 0 & 0.25 & 0.77 & 0 & 0 \\
0 & 0 & 0 & 0.23 & 0.63 & 0 \\
0 & 0 & 0 & 0 & 0.37 & 0
\end{array}\right)
$$

was used by Usher [1966] to model a Scots pine forest. A unit of time is five years. Notice that Usher has assumed that there is no loss to the population during a unit of time from any of the first five size classes so that in this application the equalities

$$
p_{i i}+p_{i+1, i}=1 \text { for } i=1,2, \ldots, m-1
$$

hold. For such models, the formula (17) simplifies to

$$
n=\sum_{i=1}^{m} \frac{f_{i i}}{1-p_{i i}} .
$$

For the matrix (18) we get from this formula the approximate value $n \simeq 15.058$. Since $n>1$ we conclude that the forest population is growing exponentially.

From Corollary 7 of the Appendix we know that $1<\lambda<n \simeq 15.058$. In fact, the dominant eigenvalue $\lambda$ of the projection matrix $A$ can be approximated numerically (as a root of the sixth degree characteristic polynomial or by other eigenvalue methods) to be $\lambda \simeq 1.2044$. This is the exponential growth rate of the population. The mean length of a generation is then $\gamma=\ln n / \ln \lambda \simeq 14.582$ time units, or 72.91 years. 
3.3. Models with a single newborn class. In this section we consider a general population model under the assumption that all newborns lie in the same class, namely class $i=1$ (without loss in generality). Thus in (5) the fertility matrix has the form (12) as in a Leslie matrix. The transition matrix $T=\left(p_{i j}\right), p_{i j} \in[0,1]$, however, is arbitrary subject to the condition (3) and assumptions (8) and (10).

The entries in the inverse $(I-T)^{-1}=\left(e_{i j}\right)$ are

$$
e_{i j}=\frac{c_{j i}}{\operatorname{det}(I-T)}
$$

where $c_{j i}$ is the cofactor of the entry $j i^{t h}$ entry in $I-T$. Then from (12) we have

$$
(I-T)^{-1} F=\frac{1}{\operatorname{det}(I-T)}\left(\begin{array}{cccc}
c_{11} f_{11} & c_{11} f_{12} & \cdots & c_{11} f_{1 m} \\
c_{12} f_{11} & c_{12} f_{12} & \cdots & c_{12} f_{1 m} \\
\vdots & \vdots & \square & \vdots \\
c_{1 m} f_{11} & c_{1 m} f_{12} & \cdots & c_{1 m} f_{1 m}
\end{array}\right) .
$$

Since each row is a scalar multiple of the first row in $F$, it follows that 0 is an eigenvalue of multiplicity $m-1$. The remaining eigenvalue and associated eigenvector of this matrix are

$$
n=\frac{1}{\operatorname{det}(I-T)} \sum_{k=1}^{m} f_{1 k} c_{1 k}
$$

and

$$
\mathbf{y}=\left(\begin{array}{c}
c_{11} \\
c_{12} \\
\vdots \\
c_{1 m}
\end{array}\right)
$$

which, if positive, implies that $n$ is the net reproductive value.

As a numerical example consider the fertility and transition matrices

$$
F=\left(\begin{array}{ccccccc}
0 & 0 & 0 & 0 & 0 & 0 & 431 \\
0 & 0 & 0 & 0 & 0 & 0 & 0 \\
0 & 0 & 0 & 0 & 0 & 0 & 0 \\
0 & 0 & 0 & 0 & 0 & 0 & 0 \\
0 & 0 & 0 & 0 & 0 & 0 & 0 \\
0 & 0 & 0 & 0 & 0 & 0 & 0 \\
0 & 0 & 0 & 0 & 0 & 0 & 0
\end{array}\right)
$$




$$
T=\left(\begin{array}{ccccccc}
0 & 0 & 0 & 0 & 0 & 0 & 0 \\
0.748 & 0 & 0 & 0 & 0 & 0 & 0 \\
0 & 0.966 & 0 & 0 & 0 & 0 & 0 \\
0.008 & 0.013 & 0.010 & 0.125 & 0 & 0 & 0 \\
0.070 & 0.007 & 0 & 0.125 & 0.238 & 0 & 0 \\
0.002 & 0.008 & 0 & 0.038 & 0.245 & 0.167 & 0 \\
0 & 0 & 0 & 0 & 0.023 & 0.750 & 0
\end{array}\right)
$$

considered by Werner and Caswell [1977] in a study of the dynamics of the perennial plant (Dipsacus sylvestrix Huds.). In this application the plants are placed into seven life cycle stages, namely, seeds, 1 year dormant seeds, 2 year dormant seeds, small rosettes, medium rosettes, large rosettes, and flowering plants. The matrix $T$ satisfies the dissipative condition (9). The net reproductive value can be calculated by the formula (19). Thus,

$$
n=\frac{1}{\operatorname{det}(I-T)} f_{17} c_{17}
$$

where $f_{17}=431, \operatorname{det}(I-T) \simeq 0.5554$ and

$$
\begin{aligned}
c_{17} & =\operatorname{det}\left(\begin{array}{cccccc}
-0.748 & 1 & 0 & 0 & 0 & 0 \\
0 & -0.966 & 1 & 0 & 0 & 0 \\
-0.008 & -0.013 & -0.01 & 0.875 & 0 & 0 \\
-0.070 & -0.007 & 0 & -0.125 & 0.762 & 0 \\
-0.002 & -0.008 & 0 & -0.038 & -0.245 & 0.833 \\
0 & 0 & 0 & 0 & -0.023 & -0.75
\end{array}\right) \\
& =1.8525 \times 10^{-2} .
\end{aligned}
$$

This yields $n \simeq 14.376$. Moreover, from (20) we find that

$$
0<\mathrm{y} \simeq\left(\begin{array}{c}
29.981 \\
22.426 \\
21.663 \\
0.8549 \\
3.1003 \\
1.2382 \\
1
\end{array}\right)
$$

is indeed positive. Since $n>1$ this population is growing exponentially. 
From Corollary 7 of the Appendix we know that $1<\lambda<n \simeq 14.376$. In fact, the dominant eigenvalue $\lambda$ of the projection matrix $A$ can be approximated numerically (as a root of the seventh degree characteristic polynomial or by other eigenvalue methods) to be $\lambda \simeq 1.8842$. This is the exponential growth rate of the population. The mean length of a generation is then $\gamma=\ln n / \ln \lambda \simeq 4.2076$ years.

3.4. A remark about general matrix models. Although the definition and results above concerning the net reproductive value $n$ were developed for non-negative projection matrices $A$ that have an additive decomposition $A=F+T$ into a fertility matrix $F \geq 0$ and a transition matrix $T \geq 0$, the results remain valid for any additive decomposition of a projection matrix $A=\widehat{F}+\widehat{T}$ for which $\widehat{F}$ and $\widehat{T}$ satisfy the assumption needed for Theorem 3 .

For example, a model of the form (5) can be formulated using a nonnegative projection matrix without reference to the fertility and transition matrices utilized above. The entries in $A$ might be numerical values statistically approximated from data (Lefkovitch [1965], Woodward [1982]) or a model of the form (5) might arise from the linearization of a nonlinear model at an equilibrium. If an additive decomposition of such an $A$ is made in a suitable manner so that Theorem 3 and Corollary 4 can be applied, then one obtains a stability criterion for the trivial equilibrium based upon the value of $n$ defined as above. This value of $n$ will not necessarily have the biological interpretation of a net reproductive value, however (because $\widehat{F}$ and $\widehat{T}$ may no longer have the interpretations of a fertility and transition matrix). With an appropriate decomposition of $A$ it might be possible to compute the value of $n$ and hence determine the stability properties of the trivial equilibrium by means of one of the formulas for $n$ given above.

For example, suppose a non-negative irreducible projection matrix that satisfies (7)

$$
A=\left(\begin{array}{cccc}
a_{11} & a_{12} & \cdots & a_{1 m} \\
a_{21} & a_{22} & \cdots & a_{2 m} \\
\vdots & \vdots & \square & \vdots \\
a_{m 1} & a_{m 2} & \cdots & a_{m m}
\end{array}\right)
$$


is additively decomposed as $A=\widehat{F}+\widehat{T}, \widehat{F} \geq 0, \widehat{T} \geq 0$ where

$$
\begin{aligned}
\widehat{F} & =\left(\begin{array}{cccc}
a_{11} & a_{12} & \cdots & a_{1 m} \\
0 & 0 & \cdots & 0 \\
\vdots & \vdots & \square & \vdots \\
0 & 0 & \cdots & 0
\end{array}\right) \\
\widehat{T} & =\left(\begin{array}{cccc}
0 & 0 & \cdots & 0 \\
a_{21} & a_{22} & \cdots & a_{2 m} \\
\vdots & \vdots & \square & \vdots \\
a_{m 1} & a_{m 2} & \cdots & a_{m m}
\end{array}\right) .
\end{aligned}
$$

We require that the inverse

$$
\widehat{E}=(I-\widehat{T})^{-1}=\left(e_{i j}\right)
$$

exists and is non-negative. The entries of $\widehat{E}$ are given by the formula

$$
e_{i j}=\frac{c_{j i}}{\operatorname{det}(I-T)}
$$

where $c_{j i}$ is the cofactor of the $j i^{\text {th }}$ entry of $I-\widehat{T}$. An easy calculation shows that

$$
(I-\widetilde{T})^{-1} \widetilde{F}=\frac{1}{\operatorname{det}(I-T)}\left(\begin{array}{cccc}
c_{11} a_{11} & c_{11} a_{12} & \cdots & c_{11} a_{1 m} \\
c_{12} a_{11} & c_{12} a_{12} & \cdots & c_{12} a_{1 m} \\
\vdots & \vdots & \square & \vdots \\
c_{1 m} a_{11} & c_{1 m} a_{12} & \cdots & c_{1 m} a_{1 m}
\end{array}\right),
$$

a matrix which is necessarily non-negative. If this matrix has a dominant eigenvalue then Theorem 3 and its Corollary 4 remain valid with $n$ taken as this dominant eigenvalue. Because each column (row) is a multiple of the same column (row) it follows that this matrix has 0 as an eigenvalue of multiplicity $m-1$. The remaining eigenvalue is given by the formula

$$
\frac{1}{\operatorname{det}(I-\widetilde{T})} \sum_{k=1}^{m} a_{1 k} c_{1 k}
$$

(The column vector $\mathrm{y}=\operatorname{col}\left(c_{1 j}\right)$ is an eigenvector.) Thus, if this quantity is nonzero and thereby the dominant eigenvalue of $(I-\widetilde{T})^{-1} \widetilde{F}$, 
then the trivial equilibrium is stable if this quantity is less than 1 in magnitude and unstable if it is greater than 1 in magnitude.

A similar decomposition using the $l^{\text {th }}$ row of $A$ for $F$ instead of the first leads, under similar assumptions, to the quantity

$$
\frac{1}{\operatorname{det}(I-T)} \sum_{k=1}^{m} a_{l k} c_{l k}
$$

by means of which stability can be determined. Of course, this dominant eigenvalue may not have the biological interpretation of the expected number of offspring per individual per life time and hence not be the net reproductive value, depending on how $A$ is additively decomposed.

4. Application to nonlinear models. When the projection matrix $A$ is constant the model equation (5) is linear. In so-called density dependent models, however, the fertility and transition rates are dependent upon population density and the projection matrix becomes a function of $\mathbf{x}(t)$. The resulting matrix equation

$$
\begin{gathered}
\mathbf{x}(t+1)=A(\mathbf{x}(t)) \mathbf{x}(t), \quad \mathbf{x}\left(t_{0}\right) \geq 0 \\
A(\mathbf{x}(t))=F(\mathbf{x}(t))+T(\mathbf{x}(t)) \geq 0 \\
t=t_{0}, t_{0}+1, t_{0}+2, \ldots
\end{gathered}
$$

is nonlinear. The stability properties of the trivial equilibrium $\mathbf{x}=0$ are of obvious importance because of their implications for the survival of the population. Local stability of the trivial equilibrium determines not only the long term survivability of populations at low density, but is related to the existence of nontrivial equilibria (Cushing [1988, 1993, 1994]).

The local stability properties of the trivial equilibrium $\mathrm{x}=0$ can be studied by means of the linearization of equation (22). The linearized equation

$$
\begin{gathered}
\mathbf{x}(t+1)=A(0) \mathbf{x}(t) \\
t=t_{0}, t_{0}+1, t_{0}+2, \ldots
\end{gathered}
$$

has a constant projection matrix and is of the form of equation (5). Therefore, Theorem 3 and its Corollary, as well as the formulas above 
for the net reproductive value associated with the linearized projection matrix $A(0)$, can be used to investigate the local stability of the trivial equilibrium. In this context, the net reproductive value of $A(0)$ will be called the inherent net reproductive value. The word "inherent" refers to the fact that this number is the expected number of offspring of an individual over its life time when the population density is low (technically, zero).

The nonlinear matrix equation (22) can also have positive equilibria. The local stability of a positive equilibrium $\mathbf{x}=\mathbf{x}^{e}>0$ can be determined from that of the linearization of (22), provided it is hyperbolic, i.e., no eigenvalue of the linearization has magnitude equal to 1 . The linearization of (22) at $\mathrm{x}^{e}$ is a linear matrix equation of the form (23) in which the coefficient matrix $A(0)$ is replaced by the Jacobian $J$ of $A(\mathbf{x}) \mathbf{x}$ evaluated at $\mathbf{x}=\mathbf{x}^{e}$. Although this linear equation may or may not have the properties of a demographic model equation, the remarks of Section 3.4 might nonetheless be applicable with regard to determining its stability properties.

4.1. A model for flour beetle dynamics. The nonlinear equations

$$
\begin{aligned}
& x_{1}(t+1)=b x_{3}(t) e^{-c_{e l} x_{2}(t)-c_{e a} x_{3}(t)} \\
& x_{2}(t+1)=\left(1-\mu_{l}\right) x_{1}(t) \\
& x_{3}(t+1)=x_{2}(t) e^{-c_{p a} x_{3}(t)}+\left(1-\mu_{a}\right) x_{3}(t)
\end{aligned}
$$

constitute the deterministic model for the dynamics of flour beetles of the genus Tribolium introduced in Dennis, et al. [1995] and Constantino, et al. [1995]. The model distinguishes three classes, namely larva $x_{1}(t)$, pupa $x_{2}(t)$, and adults $x_{3}(t)$. The exponential nonlinearities account for inter-class cannibalism. We will consider the simplified case when larval cannibalism on eggs is ignored so that $c_{e l}=0$. This case is also studied in Dennis, et al. [1995]. The resulting equations

$$
\begin{aligned}
& x_{1}(t+1)=b x_{3}(t) e^{-c_{e a} x_{3}(t)} \\
& x_{2}(t+1)=\left(1-\mu_{l}\right) x_{1}(t) \\
& x_{3}(t+1)=x_{2}(t) e^{-c_{p a} x_{3}(t)}+\left(1-\mu_{a}\right) x_{3}(t)
\end{aligned}
$$


have the a nonlinear Leslie matrix form with

$$
\begin{aligned}
& F(\mathbf{x})=\left(\begin{array}{ccc}
0 & 0 & b e^{-c_{e a} x_{3}} \\
0 & 0 & 0 \\
0 & 0 & 0
\end{array}\right), \\
& T(\mathbf{x})=\left(\begin{array}{ccc}
0 & 0 & 0 \\
1-\mu_{l} & 0 & 0 \\
0 & e^{-c_{p a} x_{3}} & 1-\mu_{a}
\end{array}\right) .
\end{aligned}
$$

The exponentials account for losses due to cannibalism, with the nonnegative coefficients $c_{e a}$ and $c_{p a}$ (not both equal to 0 ) corresponding to egg and pupa cannibalism by adults respectively. The parameter $b>0$ is the per adult larva production rate and $\mu_{l}, \mu_{a} \in(0,1)$ are the larval and adult mortality rates. The unit of time is the maturation period.

The linearized equation at the trivial equilibrium $\mathbf{x}=\left(x_{1}, x_{2}, x_{3}\right)=$ $(0,0,0)=0$ has coefficient matrix $A(0)=F(0)+T(0)$ where

$$
\begin{aligned}
& F(0)=\left(\begin{array}{lll}
0 & 0 & b \\
0 & 0 & 0 \\
0 & 0 & 0
\end{array}\right), \\
& T(0)=\left(\begin{array}{ccc}
0 & 0 & 0 \\
1-\mu_{l} & 0 & 0 \\
& 1 & 1-\mu_{a}
\end{array}\right) .
\end{aligned}
$$

From formula (19) we find that the inherent net reproductive value is

$$
n=\frac{1-\mu_{l}}{\mu_{a}} b
$$

and that

$$
\mathbf{y}=\left(\begin{array}{c}
1 \\
1-\mu_{l} \\
\left(1-\mu_{l}\right) / \mu_{a}
\end{array}\right)>0 .
$$

Theorem 3 and its Corollary 4 (together with the linearization principle) imply that the trivial equilibrium $\mathbf{x}=0$ is locally asymptotically stable if $n=\left(1-\mu_{l}\right) b / \mu_{a}<1$ and unstable if $n=\left(1-\mu_{l}\right) b / \mu_{a}>1$.

According to the general theory in Cushing [1988, 1993] there exists a global continuum of positive equilibria that bifurcates from the trivial 
equilibrium at $n=\left(1-\mu_{l}\right) b / \mu_{a}=1$. In this example these equilibria can be found analytically; they exist for $n>1$ and are given by the formulas

$$
\begin{aligned}
x_{1}^{e} & =\frac{b}{c_{e a}+c_{p a}} n^{-c_{e a} /\left(c_{e a}+c_{p a}\right)} \ln n \\
x_{2}^{e} & =\left(1-\mu_{l}\right) \frac{b}{c_{e a}+c_{p a}} n^{-c_{e a} /\left(c_{e a}+c_{p a}\right)} \ln n \\
x_{3}^{e} & =\frac{1}{c_{e a}+c_{p a}} \ln n .
\end{aligned}
$$

Moreover, it is known from the general theory that because the bifurcation is "supercritical" these equilibria are locally asymptotically stable at least for $n>1$ near 1 . A more precise local stability result can be obtained from the linearized equations at the equilibrium $\mathbf{x}^{e}=\left(x_{1}^{e}, x_{2}^{e}, x_{3}^{e}\right)$. This results in a linear matrix equation (5) with projection matrix given by the Jacobian of the right hand sides of equations (24) evaluated at $\mathbf{x}^{e}$, namely

$$
J=\left(\begin{array}{ccc}
0 & 0 & x_{1}^{e}\left(-c_{e a}+1 / x_{3}^{e}\right) \\
1-\mu_{l} & 0 & 0 \\
0 & \mu_{a} x_{3}^{e} / x_{2}^{e} & 1-\mu_{a}\left(1+c_{p a} x_{3}^{e}\right)
\end{array}\right) .
$$

This matrix is nonnegative, irreducible, and primitive (the fourth power is positive) provided the first and third entries in the third column are positive, i.e., provided the inequalities

$$
0<x_{3}^{e}<\min \left\{\frac{1}{c_{e a}}, \frac{1}{c_{p a}} \frac{1-\mu_{a}}{\mu_{a}}\right\}
$$

hold. These inequalities hold if and only the inherent net reproductive value $n$ satisfies the inequalities

$$
1<n<\min \left\{\exp (r+1), \exp \left(\left(\frac{1}{r}+1\right)\left(\frac{1}{\mu_{a}}-1\right)\right)\right\} .
$$

where $r$ is the ratio of the cannibalism coefficients

$$
r \doteq \frac{c_{p a}}{c_{e a}}>0
$$


Thus, $J$ satisfies the conditions in (7).

The matrix $J$ can can be additively decomposed into the sum $J=$ $\widehat{F}+\widehat{T}$ of nonnegative matrices

$$
\begin{aligned}
\widehat{F} & =\left(\begin{array}{ccc}
0 & 0 & x_{1}^{e}\left(-c_{e a}+1 / x_{3}^{e}\right) \\
0 & 0 & 0 \\
0 & 0 & 0
\end{array}\right) \\
\widehat{T} & =\left(\begin{array}{ccc}
0 & 0 & 0 \\
1-\mu_{l} & 0 & 0 \\
0 & \mu_{a} x_{3}^{e} / x_{2}^{e} & 1-\mu_{a}\left(1+c_{p a} x_{3}^{e}\right)
\end{array}\right)
\end{aligned}
$$

The inverse

$$
\begin{aligned}
0 & \leq(I-\widehat{T})^{-1} \\
& =\left(\begin{array}{ccc}
1 & 0 & 0 \\
1-\mu_{l} & 1 & 0 \\
-\left(-1+\mu_{l}\right) x_{3} /\left(x_{2}\left(1+c_{p a} x_{3}\right)\right) & x_{3} /\left(x_{2}\left(1+c_{p a} x_{3}\right)\right) & 1 /\left(\mu_{a}\left(1+c_{p a} x_{3}\right)\right)
\end{array}\right)
\end{aligned}
$$

exists. Thus, $J$ satisfies (8) under condition (26).

The formula (21) leads, after some algebra, to the formulas

$$
\frac{1-c_{e a} x_{3}^{e}}{1+c_{p a} x_{3}^{e}}, \quad \mathbf{y}=\left(\begin{array}{c}
1 \\
1-\mu_{l} \\
\left(x_{3}^{e} / x_{2}^{e}\left(1-\mu_{l}\right) /\left(1+c_{p a} x_{3}\right)\right)
\end{array}\right)
$$

for the dominant eigenvalue of $(I-\widehat{T})^{-1} \widehat{F}$ and its associated right eigenvector. Under condition (26) y $>0$ and this positive dominant eigenvalue is clearly less than 1 . This means $J$ satisfies (10).

It follows from Theorem 3 and Corollary 4 that the trivial equilibrium of the linearization is stable. We conclude, by the linearization principle, that the positive equilibria (25) are locally asymptotically stable when the inherent net reproductive value $n$ satisfies the inequalities (26).

4.2. A juvenile vs. adult competition model. Consider a population consisting of one juvenile and two adult size classes, one consisting of smaller and less fertile adults and the other of larger and more fertile adults. The adult size attained at maturation is determined 
by the amount of competition for resources experienced during the juvenile phase. In Cushing and $\mathrm{Li}$ [1992] the effects of this intra-specific competition was studied by means of a model of the following form

$$
\begin{aligned}
& x_{1}(t+1)=f_{1} x_{2}(t)+f_{2} x_{3}(t) \\
& x_{2}(t+1)=\phi(w(t)) x_{1}(t) \\
& x_{3}(t+1)=(1-\phi(w(t))) x_{1}(t) .
\end{aligned}
$$

Here $x_{1}, x_{2}$, and $x_{3}$ are the densities of the juvenile, smaller adults, and larger adults, respectively, and $0<f_{1}<f_{2}$ are the per capita fertilities of the smaller and larger adults, respectively. The increasing function $\phi:[0,+\infty) \rightarrow[0,1], 0<\varphi(0)<1$, gives the fraction of juveniles that mature as smaller adults as a function of the weighted total population size $w=x_{1}+w_{1} x_{2}+w_{2} x_{3}$. The coefficients $w_{i}$ measure the competitive pressure from adults on juvenile growth relative to that from juveniles themselves.

The linearization of this model at the trivial equilibrium is

$$
\begin{aligned}
& x_{1}(t+1)=f_{1} x_{2}(t)+f_{2} x_{3}(t) \\
& x_{2}(t+1)=\phi(0) x_{1}(t) \\
& x_{3}(t+1)=(1-\phi(0)) x_{1}(t)
\end{aligned}
$$

whose projection matrix $A(0)=F(0)+T(0)$ is given by

$$
\begin{aligned}
F(0) & =\left(\begin{array}{ccc}
0 & f_{1} & f_{2} \\
0 & 0 & 0 \\
0 & 0 & 0
\end{array}\right) \\
T(0) & =\left(\begin{array}{cccc}
0 & 0 & 0 \\
\phi(0) & 0 & 0 \\
1-\phi(0) & 0 & 0
\end{array}\right) .
\end{aligned}
$$

It is easy to calculate that

$$
(I-T(0))^{-1}=\left(\begin{array}{ccc}
1 & 0 & 0 \\
\phi(0) & 1 & 0 \\
1-\phi(0) & 0 & 1
\end{array}\right)
$$

exists and

$$
(I-T(0))^{-1} F(0)=\left(\begin{array}{ccc}
0 & f_{1} & f_{2} \\
0 & f_{1} \phi(0) & f_{2} \phi(0) \\
0 & f_{1}(1-\phi(0)) & f_{2}(1-\phi(0))
\end{array}\right) .
$$


This matrix $(I-T)^{-1} F$ has 0 as a double eigenvalue and the eigenvalue

$$
n=f_{1} \phi(0)+f_{2}(1-\phi(0))>0
$$

which defines the inherent net reproductive value. The right and left eigenvectors are

$$
\begin{aligned}
& y=\left(\begin{array}{c}
1 \\
\varphi(0) \\
1-\varphi(0)
\end{array}\right)>0, \\
& \mathbf{w}=\left(0, \quad f_{1}(1-\varphi(0)), \quad f_{2}(1-\varphi(0))\right) \geq 0 \text {. }
\end{aligned}
$$

Thus, by the linearization principle together with Theorem 3 and Corollary 4 , the trivial equilibrium is locally asymptotically stable if this number $n<1$ and unstable if $n>1$.

4.3. A host parasite model. Our final application deals with a model of multi-species interactions. We chose it in order to show how to use the results and techniques above in more complicated situations (in this case, a multi-species interaction). It deals with a host/parasitoid interaction and details concerning the model and its analysis can be found in (Barclay [1986], Crowe and Cushing [1994]).

Let

$$
x=\left(\begin{array}{l}
h \\
p
\end{array}\right)
$$

where

$$
\mathbf{h}=\left(\begin{array}{c}
h_{1} \\
\vdots \\
h_{m+1}
\end{array}\right), \quad \mathbf{p}=\left(\begin{array}{c}
p_{1} \\
\vdots \\
p_{w}
\end{array}\right)
$$

denote the vector $\mathbf{h}$ of $m+1$ age class densities $h_{1}, \ldots, h_{m+1}$ of a host population and the vector $\mathbf{p}$ of $w$ adult age classes densities $p_{1}, \ldots, p_{w}$ of a parasitoid. Here $h_{i}$ is the non-parasitized larva of age $i \leq m$ and $h_{m+1}$ is the number of the adult hosts.

In the absence of the parasitoid the dynamics of the host population is governed by the $(m+1) \times(m+1)$ nonlinear Leslie matrix equation 
(22) with $A(\mathbf{h})=F(\mathbf{h})+T$ where

$$
\begin{aligned}
F(\mathbf{h}) & =\left(\begin{array}{cccc}
0 & 0 & \cdots & f e^{-g h_{m}} \\
0 & 0 & \cdots & 0 \\
\vdots & \vdots & \square & \vdots \\
0 & 0 & \cdots & 0
\end{array}\right), \\
T & =\left(\begin{array}{ccccc}
0 & 0 & \cdots & 0 & 0 \\
p & 0 & \cdots & 0 & 0 \\
0 & p & \cdots & 0 & 0 \\
\vdots & \vdots & \square & \vdots & \vdots \\
0 & 0 & \cdots & p & 0
\end{array}\right)
\end{aligned}
$$

whose linearization (23) at the trivial equilibrium $\mathbf{h}=\mathbf{0}$ is a classical Leslie matrix whose net reproductive value is $n=f p^{m}$. Thus, in the absence of the parasitoid the host population survives if $f p^{m}>1$ and goes extinct if $f p^{m}<1$.

The model of the host-parasitoid interaction takes the form of (22)

$$
\mathbf{x}(t+1)=\bar{A}(\mathbf{x}(t)) \mathbf{x}(t)
$$

where the $(m+1+w) \times(m+1+w)$ projection matrix, in block form, is given by

$$
\begin{aligned}
& \bar{A}(\mathbf{x})=\bar{F}(\mathbf{x})+\bar{T}(\mathbf{x}) \\
& \bar{F}(\mathbf{x})=\left(\begin{array}{cc}
F(\mathbf{h}) & 0 \\
0 & C(\mathbf{x})
\end{array}\right), \\
& \bar{T}(\mathbf{x})=\left(\begin{array}{cc}
T(\mathbf{p}) & 0 \\
0 & D
\end{array}\right)
\end{aligned}
$$

where the $(m+1) \times(m+1)$ host transition matrix $T$ has been modified to account for parasitization at the $a^{\text {th }}$ larval stage as follows

$$
T(\mathbf{p})=\left(\begin{array}{ccccccc}
0 & 0 & \cdots & 0 & \cdots & 0 & 0 \\
p & 0 & \cdots & 0 & \cdots & 0 & 0 \\
0 & p & \cdots & 0 & \cdots & 0 & 0 \\
\vdots & \vdots & \square & \vdots & \square & \vdots & \vdots \\
0 & 0 & \cdots & p e^{-q \pi(\mathbf{p})} & \cdots & 0 & 0 \\
\vdots & \vdots & \square & \vdots & \square & \vdots & \vdots \\
0 & 0 & \cdots & 0 & \cdots & p & 0
\end{array}\right) \leftarrow(a+1)^{\text {st }} \text { row }
$$


and the $w \times w$ fertility and transition matrices, $C$ and $D$, for the parasitoid population are given by

$$
\begin{aligned}
C(\mathbf{x}) & =\frac{1-e^{-q \pi(\mathbf{p})}}{\pi(\mathbf{p})} u^{n_{0}-k} p^{k} h_{a}\left(\begin{array}{cccc}
1 & 1 & \cdots & 1 \\
0 & 0 & \cdots & 0 \\
\vdots & \vdots & \square & \vdots \\
0 & 0 & \cdots & 0
\end{array}\right) \\
D & =\left(\begin{array}{ccccc}
0 & 0 & \cdots & 0 & 0 \\
s & 0 & \cdots & 0 & 0 \\
\vdots & \vdots & \square & \vdots & \vdots \\
0 & 0 & \cdots & s & 0
\end{array}\right) .
\end{aligned}
$$

The various coefficients appearing in these matrices are: $a$ is the age of parasitized larva, $p$ is the survivability of non-parasitized hosts, $f$ is adult host fertility, $g$ is the density coefficient for adult hosts, $q$ is the constant related to search efficiency, $s$ is the survivability of adult parasites, $n_{0}$ is the development time for parasites, $k$ is the time after which parasitized affect host mortality $\left(\leq n_{0}\right), u$ is the survivability of parasitized hosts after $k$ time units and $\pi(\mathbf{p})=\sum_{i=1}^{w} p_{i}$ is the total adults parasite numbers.

First we consider the stability of the trivial solution $\mathrm{x}=0$ in which both populations are absent. Although the interpretation of an inherent net reproductive value is not appropriate for this two species model, the dominant eigenvalue of $(I-\bar{T}(0))^{-1} \bar{F}(0)$ can still be used to determine the stability properties of the trivial solution. Since

$$
(I-\bar{T}(0))^{-1} \bar{F}(0)=\left(\begin{array}{cc}
(I-T(0))^{-1} F(0) & 0 \\
0 & 0
\end{array}\right)
$$

it follows that this dominant eigenvalue is in fact the inherent net reproductive value $n=f p^{m}$ of the isolated host.

Thus, the trivial solution of the host-parasitoid interaction is stable (and both species go extinct) if the trivial solution of the isolated host population is stable, i.e., $n<1$. It makes sense, of course, that both the host and the parasitoid should go extinct if the host population cannot survive in absence of the parasitoid.

In order to make a last point concerning the methods developed above for determining stability we consider the case when there exists 
nontrivial, non-negative equilibria. If the host is viable in the absence of the parasitoid, i.e., $n>1$, such an equilibrium will exist and the species will not both go extinct. If $n>1$ then there are nontrivial equilibria

$$
\mathbf{x}^{e}=\left(\begin{array}{c}
\mathbf{h}^{e} \\
0
\end{array}\right)
$$

where

$$
\mathrm{h}^{e}=\frac{p^{1-m}}{g} \ln (n)\left(\begin{array}{c}
1 \\
p \\
\vdots \\
p^{m}
\end{array}\right) .
$$

The linearization of (27) at $\mathbf{x}^{e}$ is

$$
\left(\begin{array}{l}
\mathrm{h}(t+1) \\
\mathrm{p}(t+1)
\end{array}\right)=\left(\begin{array}{cc}
B+T(0) & 0 \\
0 & D
\end{array}\right)\left(\begin{array}{l}
\mathrm{h}(t) \\
\mathbf{p}(t)
\end{array}\right)
$$

where $B$ is the $(m+1) \times(m+1)$ matrix

$$
B=\left(\begin{array}{cccc}
0 & \cdots & -g f e^{-g x_{m}^{e}} x_{m+1}^{e} & f e^{-g x_{m}^{e}} \\
0 & \cdots & 0 & 0 \\
\vdots & \square & \vdots & \vdots \\
0 & \cdots & 0 & 0
\end{array}\right) .
$$

One entry in the matrix $B$ is negative and therefore $B$ (and hence the linearized projection matrix) are not non-negative. This means that Theorem 3 and Corollary 4 cannot be applied to determine (local) stability of the nontrivial equilibrium. However, except for this nonnegativity, the linearization has a Leslie matrix model form and a direct calculation yields the dominant eigenvalue

$$
\lambda=(1-\ln (n))^{1 /(m+1)} .
$$

We also obtain from (14) the dominant eigenvalue

$$
\eta=1-\ln (n)
$$

of $(I-\bar{T})^{-1} \bar{F}\left(\mathbf{x}^{e}\right)$. Hence, $|\lambda|<1$ if and only if $|\eta|<1$. The equilibrium $\left(\mathbf{x}^{e}, 0\right)$ is stable if $|\eta|<1$ and unstable if $|\eta|>1$. 
This last result demonstrates the dominant eigenvalue of the matrix $(I-T)^{-1} F$ can be used to determine the stability even when the conditions of the Theorem 3 and its Corollary do not hold.

5. Concluding remarks. In this paper we have given a mathematical definition of the net reproductive value $n$ for a very general class of linear matrix population dynamical models. This number has the biological interpretation of the expected number of offspring produced by an individual over the course of its life. Our mathematical definition provides a generalization of the familiar formula for the net reproductive value in a Leslie age-structured population model. It is biologically intuitive that the exponential growth of a population is possible if and only if $n>1$ and that the population dies out exponentially if and only if $n<1$. These stability properties of the zero state equilibrium are well known for Leslie matrices. For general structured models it is not mathematically obvious, however, that these facts are valid for general linear matrix models. The main result of this paper (contained in Theorem 1 and Corollary 4) is that these facts are indeed valid for a very general class of linear matrix models.

Under suitable assumptions on the projection matrix, a population described by a linear matrix model will exponentially die out, exponentially grow, or remain in equilibrium if the dominant eigenvalue $\lambda$ is less than, greater than, or equal to 1 respectively. Theorem 1 proves that the same is true if the net reproductive value $n$ is less than, greater than, or equal to 1 respectively. Thus, the asymptotic dynamics of such a population can be determined by a single quantity, either $\lambda$ or $n$. All parameters in a matrix model (of which there can be a large number) contribute to the dynamics of the population in that they contribute to the calculation of $\lambda$ or $n$. Determining exactly how a model parameter contributes to the calculation of $\lambda$ and $n$ can be a complicated algebraic problem, however.

In general there is no algebraic formula for the dominant eigenvalue $\lambda$ in terms of the entries in the projection matrix (it is the dominant root of a high degree characteristic polynomial). On the other hand, we have shown above that it is often the case that an explicit formula relating $n$ to the entries of the projection matrix can be found. This not only permits the convenient numerical calculation of the net reproductive value $n$ directly from numerical entries in the projection matrix, but 
it allows general parameter studies to be made on the effects that individual entries have on $n$ and hence the long term asymptotic dynamics of the population. Without such explicit formulas, this is more difficult to do for the dominant eigenvalue $\lambda$. An example of the importance of such studies can be found in the sensitivity analysis of Caswell [1989]. This analysis relates changes in model parameters to changes in the dominant eigenvalue $\lambda$ and uses the results to study problems in adaptation and evolutionary demography. It would be interesting to perform such investigations using $n$ in place of the dominant eigenvalue $\lambda$. Indeed, given that the net reproductive value $n$ is a "fitness" measure of an individual it might be more meaningful to use it in such studies rather than the total population growth rate $\lambda$ (Caswell, personal communication).

We have shown that both $\lambda$ and $n$ can be used to determine the asymptotic dynamics of linear matrix models (and, in our examples, in some nonlinear models as well). Moreover, we have pointed out some of the advantages of using $n$ for this purpose. It must be kept in mind, however, that while both parameters can be used to determine asymptotic dynamics, they measure different properties of the population's dynamics. In particular, the net reproductive value $n$ does not measure the growth rate of the total population. To determine this growth rate the dominant eigenvalue $\lambda$ must be calculated. Although our analysis resulted in some inequalities relating $\lambda$ and $n$, we have no formula that directly relates these two quantities.

The net reproductive value has also been useful, as a bifurcation parameter, in the study of general nonlinear matrix models (Cushing $[1988,1993,1994])$. We saw something of this in the examples above, and see the potential for its use in future studies.

\section{APPENDIX}

LEMMA 5. Let $A$ satisfy (7). If $(T \mathbf{y})_{i}=\sum_{j=1}^{m} p_{i j} y_{j}$, where $\mathbf{y}$ is the positive unit eigenvector of $(I-T)^{-1} F$ corresponding to $n$, then

$$
\min _{i} \frac{(T \mathrm{y})_{i}}{y_{i}}<1, \quad \max _{i} \frac{(T \mathrm{y})_{i}}{y_{i}} \leq 1
$$


PROOF. From

$$
(I-T)^{-1} F \mathbf{y}=n \mathbf{y}
$$

follows

$$
T \mathrm{y}=\mathrm{y}-\frac{1}{n} F \mathrm{y}
$$

and

$$
\frac{(T \mathbf{y})_{i}}{y_{i}}=1-\frac{1}{n} \frac{(F \mathbf{y})_{i}}{y_{i}}
$$

from which we get that

$$
\begin{aligned}
\min _{i} \frac{(T \mathbf{y})_{i}}{y_{i}} & =1-\max _{i} \frac{1}{n} \frac{(F \mathbf{y})_{i}}{y_{i}} \\
\max _{i} \frac{(T \mathbf{y})_{i}}{y_{i}} & =1-\min _{i} \frac{1}{n} \frac{(F \mathbf{y})_{i}}{y_{i}} .
\end{aligned}
$$

Because $F \geq 0$, but $F \neq 0$, and because $\mathrm{y}>0$, we have that

$$
\max _{i} \frac{1}{n} \frac{(F \mathbf{y})_{i}}{y_{i}}>0 \text { and } \min _{i} \frac{1}{n} \frac{(F \mathbf{y})_{i}}{y_{i}} \geq 0
$$

from which the desired inequalities follow.

PROOF OF THEOREM 3. We begin with a proof of Theorem 3 when both $A$ and $R \doteq(I-T)^{-1} F$ are irreducible. Note that

$$
\begin{aligned}
& A=(I-T) R+T, \\
& R=(I-T)^{-1}(A-T) .
\end{aligned}
$$

The positive unit eigenvalues and their eigenvectors satisfy

$$
A \mathbf{v}=\lambda \mathbf{v}, \quad R \mathbf{y}=n \mathbf{y}
$$

and the eigenvalues have the characterizations

$$
\begin{aligned}
& \lambda=\max _{\mathbf{x} \geq 0} \min _{i, x_{i} \neq 0} \frac{(A \mathbf{x})_{i}}{x_{i}}=\min _{\mathbf{x} \geq 0} \max _{i, x_{i} \neq 0} \frac{(A \mathbf{x})_{i}}{x_{i}} \\
& n=\max _{\mathbf{x} \geq 0} \min _{i, x_{i} \neq 0} \frac{(R \mathbf{x})_{i}}{x_{i}}=\min _{\mathbf{x} \geq 0} \max _{i, x_{i} \neq 0} \frac{(R \mathbf{x})_{i}}{x_{i}}
\end{aligned}
$$


where $(A \mathbf{x})_{i}=\sum_{j=1}^{m} a_{i j} x_{j}$, etc., (Gantmacher [1959]).

From (28) we have

$$
\begin{aligned}
& A \mathbf{y}=n \mathbf{y}+(1-n) T \mathbf{y} \\
& R \mathbf{v}=\lambda \mathbf{v}+(\lambda-1)(I-T)^{-1} T \mathbf{v}
\end{aligned}
$$

and hence

$$
\begin{aligned}
& \frac{(A \mathbf{y})_{i}}{y_{i}}=n+(1-n) \frac{(T \mathbf{y})_{i}}{y_{i}} \\
& \frac{(R \mathbf{v})_{i}}{v_{i}}=\lambda+(\lambda-1) \frac{\left((I-T)^{-1} T \mathbf{v}\right)_{i}}{v_{i}} .
\end{aligned}
$$

These equalities, together with the min-max and max-min characterizations above, imply the inequalities

$$
\begin{aligned}
& \lambda \geq n+\min _{i}(1-n) \frac{(T \mathbf{y})_{i}}{y_{i}} \\
& \lambda \leq n+\max _{i}(1-n) \frac{(T \mathbf{y})_{i}}{y_{i}}
\end{aligned}
$$

for $\lambda$ and

$$
\begin{aligned}
& n \geq \lambda+\min _{i}(\lambda-1) \frac{\left((I-T)^{-1} T \mathbf{v}\right)_{i}}{v_{i}} \\
& n \leq \lambda+\max _{i}(\lambda-1) \frac{\left((I-T)^{-1} T \mathbf{v}\right)_{i}}{v_{i}}
\end{aligned}
$$

for $n$. These inequalities imply the following facts:

$$
\begin{aligned}
& n<1 \Rightarrow n \leq \lambda \leq n+(1-n) \max _{i} \frac{(T \mathbf{y})_{i}}{y_{i}} \\
& n>1 \Rightarrow n+(1-n) \max _{i} \frac{(T \mathbf{y})_{i}}{y_{i}} \leq \lambda \leq n, \\
& n=1 \Rightarrow \lambda=1 .
\end{aligned}
$$

In a similar fashion we get

$$
\begin{aligned}
& \lambda<1 \Rightarrow n \leq \lambda+(\lambda-1) \min _{i} \frac{\left((I-T)^{-1} T \mathbf{v}\right)_{i}}{v_{i}} \\
& \lambda>1 \Rightarrow \lambda+(\lambda-1) \min _{i} \frac{\left((I-T)^{-1} T \mathbf{v}\right)_{i}}{v_{i}} \leq n \\
& \lambda=1 \Rightarrow n=1
\end{aligned}
$$


First, it is clear from these facts that $n=1$ if and only if $\lambda=1$.

Suppose that $n>1$. Then $\lambda \neq 1$. From (30) and Lemma 5 follows $\lambda \geq 1$ and hence $\lambda>1$. Conversely, suppose that $\lambda>1$. Then $n \neq 1$. From (32) follows $n \geq 1$ and hence $n>1$. This shows that $n>1$ if and only if $\lambda>1$.

From (29), (31), and Lemma 5 it follows immediately that $n<1$ if and only if $\lambda<1$.

The general case when not both $A$ and $R$ are irreducible can be proved using the fact that an arbitrary nonnegative matrix is always the limit of a sequence of irreducible, positive matrices (Gantmacher [1959]) and Lemma 6 below. These details will be omitted.

LEMMA 6. If $C \geq 0$ is a nonnegative matrix and $r \geq 0$ is a nonnegative dominant eigenvalue, then

$$
r=\max _{\mathbf{x} \geq 0} \min _{i, x_{i} \neq 0} \frac{(C \mathbf{x})_{i}}{x_{i}}=\min _{\mathbf{x} \geq 0} \max _{i, x_{i} \neq 0} \frac{(C \mathbf{x})_{i}}{x_{i}}
$$

where $(C \mathbf{x})_{i}=\sum_{j=1}^{m} c_{i j} x_{j}$.

Proof. For $k=1,2,3, \ldots$ define the matrices $C^{(k)}=\left(c_{i j}^{(k)}\right)$ where

$$
c_{i j}^{(k)}= \begin{cases}c_{i j} & \text { if } c_{i j}>0 \\ \frac{1}{k} & \text { if } c_{i j}=0 .\end{cases}
$$

It is obvious that $\lim _{k \rightarrow \infty} C^{(k)}=C$.

Since $C^{(k)}>0$ is strictly positive it follows that it is reducible and hence from Frobenius' theorem it has a strictly dominant, positive, simple eigenvalue $r_{k}>0$ which is associated with a positive unit eigenvector $\mathbf{y}^{(k)}=\left(w_{i}^{(k)}\right)$. Moreover, $r_{k}$ can be characterized in two ways as follows (Gantmacher [1959, p. 65]):

$$
r_{k}=\max _{\mathbf{x} \geq 0} \min _{i, x_{i} \neq 0} \frac{\left(C^{(k)} \mathbf{x}\right)_{i}}{x_{i}}
$$

and

$$
r_{k}=\min _{\mathbf{x} \geq 0} \max _{i, x_{i} \neq 0} \frac{\left(C^{(k)} \mathbf{x}\right)_{i}}{x_{i}} .
$$


Let $\mathbf{u} \geq 0$ be the nonnegative unit eigenvector associated with the dominant eigenvalue $r$ of the matrix $C$. From $C \mathbf{u}=r \mathbf{u}$ follows

$$
r=\frac{(C \mathbf{u})_{i}}{u_{i}} \quad \text { for all } i \text { for which } u_{i} \neq 0
$$

and

$$
r=\min _{i, w_{i} \neq 0} \frac{(C \mathbf{u})_{i}}{u_{i}}=\max _{i, w_{i} \neq 0} \frac{(C \mathbf{u})_{i}}{u_{i}}
$$

which in turns implies that

$$
r \leq \max _{\mathbf{x} \geq 0} \min _{i, x_{i} \neq 0} \frac{(C \mathbf{x})_{i}}{x_{i}} .
$$

Suppose now, for the purpose of contradiction, that

$$
r<\max _{\mathbf{x} \geq 0} \min _{i, x_{i} \neq 0} \frac{(C \mathbf{x})_{i}}{x_{i}} .
$$

Then there would exist a $\mathbf{x}^{0} \geq 0$, such that

$$
r<\min _{i, x_{i}^{0} \neq 0} \frac{\left(C \mathbf{x}^{0}\right)_{i}}{x_{i}^{0}} .
$$

Define

$$
\varepsilon \doteq \min _{i, x_{i}^{0} \neq 0} \frac{\left(C \mathbf{x}^{0}\right)_{i}}{x_{i}^{0}}-r>0 .
$$

Since $\lim _{k \rightarrow \infty} C^{(k)}=C$ and $\lim _{k \rightarrow \infty} r_{k}=r$, there exists an integer $K_{0}>0$ such that

$$
\left|\min _{i, x_{i}^{0} \neq 0} \frac{\left(C \mathbf{x}^{0}\right)_{i}}{x_{i}^{0}}-\min _{i, x_{i}^{0} \neq 0} \frac{\left(C^{(k)} \mathbf{x}^{0}\right)_{i}}{x_{i}^{0}}\right|<\frac{\varepsilon}{4}
$$

and

$$
\left|r-r_{k}\right|<\frac{\varepsilon}{4}
$$

for $k>K_{0}$. From these inequalities follow, for $k>K_{0}$,

$$
\begin{aligned}
\min _{i, x_{i}^{0} \neq 0} \frac{\left(C^{(k)} \mathbf{x}^{0}\right)_{i}}{x_{i}^{0}}>\min _{i, x_{i}^{0} \neq 0} \frac{\left(C \mathbf{x}^{0}\right)_{i}}{x_{i}^{0}}-\frac{\varepsilon}{4} \\
r_{k}<r+\frac{\varepsilon}{4}
\end{aligned}
$$


and hence

$$
\min _{i, x_{i}^{0} \neq 0} \frac{\left(C^{(k)} \mathbf{x}^{0}\right)_{i}}{x_{i}^{0}}-r_{k}>\min _{i, x_{i}^{0} \neq 0} \frac{\left(C \mathbf{x}^{0}\right)_{i}}{x_{i}^{0}}-\frac{\varepsilon}{4}-\left(r+\frac{\varepsilon}{4}\right)=\frac{\varepsilon}{2}
$$

or

$$
r_{k}<\min _{i, x_{i}^{0} \neq 0} \frac{\left(C^{(k)} \mathbf{x}^{0}\right)_{i}}{x_{i}^{0}}-\frac{\varepsilon}{2}
$$

This final inequality contradicts (33) and thus implies that the strict inequality (37) cannot be true. From (36) we obtain the desired first equality of the Lemma. The second equality can be proved in a similar fashion by using (34) and (35).

COROLlary 7 . Let $A$ satisfy (7). Then

$$
\begin{aligned}
& n<1 \Rightarrow n \leq \lambda \leq 1 \\
& n>1 \Rightarrow 1 \leq \lambda \leq n .
\end{aligned}
$$

As a final note we would point out that the irreducibility of the matrix $A$ does not in general imply the irreducibility of the matrix $R$. This can be seen from a simple two-dimensional example. Let

$$
F=\left(\begin{array}{ll}
f_{11} & 0 \\
f_{21} & 0
\end{array}\right), \quad T=\left(\begin{array}{ll}
p_{11} & p_{12} \\
p_{21} & p_{22}
\end{array}\right)
$$

with $f_{11}>0, f_{21}>0$, and $p_{i j}>0$. Then

$$
A=\left(\begin{array}{ll}
f_{11}+p_{11} & p_{12} \\
f_{21}+p_{21} & p_{22}
\end{array}\right)
$$

is irreducible, but

$$
R=\frac{1}{\left(1-p_{11}\right)\left(1-p_{22}\right)-p_{12} p_{21}}\left(\begin{array}{cc}
\left(1-p_{22}\right) f_{11}+p_{12} f_{21} & 0 \\
p_{21} f_{11}+\left(1-p_{11}\right) f_{21} & 0
\end{array}\right)
$$

is reducible. 


\section{REFERENCES}

Hugh Barclay [1986], Models of host-parasitoid interactions to determine the optimal instar of parasitization for pest control, Natur. Resource Modeling 1 , 81-104.

Hal Caswell [1989], Matrix population models: Construction, analysis, and interpretation, Sinauer Associates Inc., Sunderland, Massachusetts.

R.F. Costantino, J.M. Cushing, Brian Dennis, Robert A. Desharnais [1995], Experimentally induced transitions in the dynamic behaviour of insects, Nature $375,227-230$.

Kathleen M. Crowe and J.M. Cushing [1994], Optimal instar parasitization in a stage structured host-parasitoid model, Natur. Resource Modeling 8, 119-138.

J.M. Cushing [1988], Nonlinear matrix models and population dynamics, Natural Resource Modeling 2, 539-580.

J.M. Cushing [1993], Nonlinear matrix models for structured populations, Proc. 3rd International Conf. on Pop. Dynamics, to appear.

J.M. Cushing [1994], Nonlinear matrix equations and population dynamics, to appear.

J.M. Cushing and Jia Li [1992], Intra-specific competition and density dependent juvenile growth, Bull. Math. Biol. 54, 503-519.

Brian Dennis, Robert A. Desharnais, J.M. Cushing, and R.F. Costantino [1995], Nonlinear demographic dynamics: Mathematical models, statistical methods, and biological experiments, Ecol. Monogr., to appear.

F.R. Gantmacher [1959], Application of the theory of matrices, Interscience Publishers Inc., New York.

Wayne M. Getz and Robert G. Haight [1989], Population harvesting: Demographic models of fish, forest, and animal resources, Monographs in Population Biology 27, Princeton University Press, Princeton, New Jersey.

John Impagliazzo [1985], Deterministic aspects of mathematical demography, Biomathematics 13, Springer-Verlag, Berlin.

N. Keyfitz [1985], Applied mathematical demography, Springer, Berlin.

L.P. Lefkovitch [1965], The study of population growth in organisms grouped by stages, Biometrics 21, 1-18.

P.H. Leslie [1948], Some further notes on the use of matrices in population mathematics, Biometrika 35, 213-245.

E.G. Lewis [1942], On the generation and growth of population, Sankhya 6, 93-96.

Colin Newell [1988], Methods and models in demography, The Guilford Press, New York.

David Smith and Nathan Keyfitz [1977], Mathematical demography, Springer, Berlin.

Song Jian and Yu Jingyuan [1988], Population system control, China Academic Publishers, Beijing, and Springer, Berlin.

M.B. Usher [1966], A matrix approach to the management of renewable resources with special reference to forest, J. of Appl. Ecology 3, 355-367. 
M.B. Usher [1969], A matrix model for forest management, Biometrics 25, 309-315.

M.B. Usher [1972], Developments in the Leslie matrix model, appearing in Mathematical models in ecology (J.M.R. Jeffers, ed.), Blackwell Scientific Publishers, London, 29-60.

P.A. Werner and H. Caswell [1977], Population growth rates and age versus stagedistribution models for teasel (Dipsacus sylvestrix Huds.), Ecology 58, 1103-1111.

I.O. Woodward [1982], Modeling population growth in age-grouped organisms: $A$ simple extension to the Leslie model, Australian J. of Ecology 7, 389-394. 